Bull. Austral. Math. Soc.

$46 \mathrm{~s} 10,47 \mathrm{~s} 10,11 \mathrm{~s} 40$

VoL. $70(2004) \quad[73-86]$

\title{
PSEUDO-DIFFERENTIAL EQUATIONS CONNECTED WITH $p$-ADIC FORMS AND LOCAL ZETA FUNCTIONS
}

\author{
W.A. Zuniga-GaLindo
}

We study the asymptotics of fundamental solutions of $p$-adic pseudo-differential equations of type

$$
(f(\partial, \beta)+\lambda) u=g,
$$

where $f(\partial, \beta)$ is a pseudo-differential operator with symbol $|f|_{K}^{\beta}, \beta>0, f$ is a form of arbitrary degree with coefficients in a $p$-adic field, $\lambda \geqslant 0$, and $g$ is a Schwartz-Bruhat function.

\section{INTRODUCTION}

Let $K$ be a $p$-adic field, that is, $\left[K: \mathbb{Q}_{p}\right]<\infty$. Let $R_{K}$ be the valuation ring of $K, P_{K}$ the maximal ideal of $R_{K}$, and $\bar{K}=R_{K} / P_{K}$ the residue field of $K$. The cardinality of $\bar{K}$ is denoted by $q$. For $z \in K, v(z) \in \mathbb{Z} \cup\{+\infty\}$ denotes the valuation of $z,|z|_{K}=q^{-v(z)}$ and ac $z=z \mathfrak{p}^{-v(z)}$ where $\mathfrak{p}$ is a fixed uniformising parameter for $R_{K}$. For $x=\left(x_{1}, \ldots, x_{n}\right) \in K^{n}$ we put $\|x\|_{K}=\max _{1 \leqslant i \leqslant n}\left|x_{i}\right|_{K}$.

We denote by $S\left(K^{n}\right)$ the $\mathbb{C}$-vector space of Schwartz-Bruhat functions over $K^{n}$. The dual space $S^{\prime}\left(K^{n}\right)$ is the space of distributions over $K^{n}$. Let $f=f(x) \in K[x]$, $x=\left(x_{1}, \ldots, x_{n}\right)$, be a non-constant polynomial, and $\beta$ a positive real number. A $p$-adic pseudo-differential operator $f(\partial, \beta)$, with symbol $|f|_{K}^{\beta}$, is an operator of the form

$$
\begin{array}{rlc}
f(\partial, \beta): \mathcal{S}\left(K^{n}\right) & \rightarrow & \mathcal{S}\left(K^{n}\right) \\
\Phi & \rightarrow \mathcal{F}^{-1}\left(|f|_{K}^{\beta} \mathcal{F}(\Phi)\right),
\end{array}
$$

where $\mathcal{F}$ is the Fourier transform. The operator $f(\partial, \beta)$ is continuous and has selfadjoint extension with dense domain in $L^{2}\left(K^{n}\right)$. This operator is considered to be a $p$-adic analogue of a linear partial differential operator with constant coefficients. The $p$-adic pseudo-differential equation

$$
f(\partial, \beta) u=g, g \in \mathcal{S}\left(K^{n}\right),
$$

Received 15th December, 2003

The author thanks the Research and Grants Council of Barry University for financial support.

Copyright Clearance Centre, Inc. Serial-fee code: 0004-9727/04 \$A2.00+0.00. 
is naturally associate to $f(\partial, \beta)$. The theory of $p$-adic pseudo-differential equations is emerging motivated by the use of $p$-adic models in physics $[11,19]$. The state of the art of the theory of $p$-adic pseudo-differential operators is exposed in a recent book by Kochubei [11]. The simplest possible operator has symbol $|x|_{K}^{\beta}, \beta>0$. Vladimirov studied extensively this class of operators showing, among other results, the existence of fundamental solutions $[18,19]$. Kochubei showed the existence of fundamental solutions for elliptic operators, that is, for operators with symbols of the form $\left|f\left(x_{1}, \ldots, x_{n}\right)\right|_{K}^{\beta}$, $\beta>0$, where $f\left(x_{1}, \ldots, x_{n}\right)$ is a quadratic form satisfying $f\left(x_{1}, \ldots, x_{n}\right) \neq 0$ when $\left|x_{1}\right|_{K}$ $+\cdots+\left|x_{n}\right|_{K} \neq 0,[11,12]$. In [10] Khrennikov considered spaces of functions and distributions defined outside the singularities of a symbol, in this situation he showed the existence of a fundamental solution for a $p$-adic pseudo-differential equation with symbol $a(\xi) \neq 0$. In a recent note [21] the author observed that Atiyah's proof [1] for the existence of a fundamental solution for a linear partial differential operator with constant coefficients can be adapted to the $p$-adic case. In this proof the meromorphic continuation of the Igusa local zeta function plays a central role. On the other hand, Jang [9] and Sato [16] showed explicitly a connection between the local zeta function of a quadratic form and the $p$-adic Green function $G_{\lambda}$ (a fundamental solution) of the following pseudo-differential equation

$$
(f(\partial, \beta)+\lambda) u=g, \lambda>0, g \in \mathcal{S}\left(K^{n}\right),
$$

when $\beta=1$, and $f$ is a relative invariant of some prehomogeneous vector space. In [16] Sato showed that the asymptotics of $G_{\lambda}$, as $|f|_{K} \rightarrow \infty$, is controlled by the gamma factor of the functional equation of the local zeta function associated to $f$. Previously, Kochubei $[13,14]$, had described the asymptotics of $G_{\lambda}$, at the infinity and at the origin, when $f(\partial, \beta)$ is an elliptic operator.

This paper is dedicated to the study of the asymptotics of fundamental solutions for (1.1) and (1.2) when $f$ is a homogeneous polynomial in an arbitrary number of variables. We construct fundamental solutions "at infinity and at the origin" for (1.1), that is, solutions of (1.1) when $g$ is the characteristic function of a ball around the infinity, respectively, around the origin (see Theorem 3.1, and Corollary 3.1). The proof of Theorem 3.1 uses resolution of singularities [4], and some ideas developed by Atiyah for solving the problem of division of a distribution by an analytic function [1]. The techniques used in the proof of Theorem 3.1 allow us to establish a functional equation for the distributions of type $\chi(\operatorname{ac} f)|f|_{K}^{s}$ (see Theorem 2.2) on a certain subspace of $\mathcal{S}\left(K^{n}\right)$. Functional equations for distributions of type $\chi($ ac $f)|f|_{K}^{s}$ have been obtained by Igusa [8], Sato [15], Gyoja [3], and Denef and Meuser [2].

We also give the asymptotic expansion of the Green function $G_{\lambda}(x)$ as $\|x\|_{K} \rightarrow \infty$ (see Theorem 4.1). The proof of this result uses the technique of integration on the fibers and some properties of the Igusa local zeta function. Kochubei studied the asymptotics of the Green functions associated with elliptic operators [11, Section 2.8], [13, 14], at 
infinity and at the origin. The asymptotics obtained by Kochubei at infinity can be recovered from Theorem 4.1.

\section{PreLiminaries}

Let $\Psi: K \rightarrow \mathbb{C}^{\times}$be the additive character defined by

$$
\begin{aligned}
& \Psi: K \rightarrow \mathbb{Q}_{p} \rightarrow \mathbb{Q}_{p} / \mathbb{Z}_{p} \rightarrow \mathbb{C}^{\times} \\
& x \rightarrow \operatorname{Tr}_{K / \mathbb{Q}_{p}}(x) \quad y \quad \rightarrow \exp (2 \pi i y),
\end{aligned}
$$

where $\operatorname{Tr}_{K / \mathbb{Q}_{p}}$ is the trace mapping. Let $|d x|$ denote the Haar measure on $K^{n}$ normalised such that $\operatorname{vol}\left(R_{K}^{n}\right)=1$. We shall identify the $n$-dimensional $K$-vector space $K^{n}$ with its dual vector space via the standard inner product

$$
[x, y]=\sum_{i=1}^{n} x_{i} y_{i}, x, y \in K^{n} .
$$

The Haar measure $|d x|$ is autodual with respect the pairing $\Psi([x, y])$. For $\Phi \in S\left(K^{n}\right)$, the Fourier transform $\mathcal{F} \Phi$ of $\Phi$ is defined by

$$
(\mathcal{F} \Phi)(\xi)=\int_{K^{n}} \Phi(x) \Psi(-[x, \xi])|d x| .
$$

The Fourier transform induces a linear isomorphism of $S\left(K^{n}\right)$ onto itself, and the inverse transform is given by

$$
\Phi(x)=\int_{K^{\mathfrak{n}}}(\mathcal{F} \Phi)(\xi) \Psi([x, \xi])|d \xi| .
$$

The Fourier transform can be extended to an isometry of $L^{2}\left(K^{n}\right)$ onto $L^{2}\left(K^{n}\right)$.

We denote by $S^{\prime}\left(K^{n}\right)$ the dual space of $S\left(K^{n}\right)$, that is, the space of complex valued distributions on $K^{n}$. If $T \in S^{\prime}\left(K^{n}\right)$, we denote by $\mathcal{F} T$ its Fourier transform, that is the distribution $\langle\mathcal{F} T, \Phi\rangle=\langle T, \mathcal{F} \Phi\rangle$.

2.1. The Riesz Kernel. We shall collect some well-known results about the Riesz kernel that will be used in the next sections $[17,19]$.

The $p$-adic Gamma function $\Gamma_{n}(\alpha)$ is defined as follows

$$
\Gamma_{n}(\alpha)=\frac{1-q^{\alpha-n}}{1-q^{-\alpha}}, \alpha \in \cdot \mathbb{C}, \alpha \neq 0 .
$$

The Gamma function is meromorphic with simple zeros at $n+(2 \pi i) /(\log q) Z$ and unique simple pole at $\alpha=0$. In addition, it satisfies

$$
\Gamma_{n}(\alpha) \Gamma_{n}(n-\alpha)=1, \alpha \notin\{0\} \cup\left\{n+\frac{2 \pi i}{\log q} z ; \dot{z} \in \mathbb{Z}\right\} .
$$


The Riesz kernel $R_{\alpha}$ is the distribution determined by the function

$$
\mathcal{R}_{\alpha}(x)=\frac{\|x\|_{K}^{\alpha-n}}{\Gamma_{n}(\alpha)}, x \in K^{n}, \operatorname{Re}(\alpha)>0, \alpha \notin n+\frac{2 \pi i}{\log q} \mathbb{Z} .
$$

The Riesz kernel possesses, as a distribution, a meromorphic continuation to $\mathbb{C}$ given by

$$
\begin{aligned}
\left\langle\mathcal{R}_{\alpha}, \Phi\right\rangle=\Phi(0)\left(\frac{1-q^{-n}}{1-q^{\alpha-n}}\right)+\left(\frac{1-q^{-\alpha}}{1-q^{\alpha-n}}\right) & \int_{\|x\|_{K} \leqslant 1}(\Phi(x)-\Phi(0))\|x\|_{K}^{\alpha-n}|d x| \\
& +\left(\frac{1-q^{-\alpha}}{1-q^{\alpha-n}}\right) \int_{\|x\|_{K}>1} \Phi(x)\|x\|_{K}^{\alpha-n}|d x|
\end{aligned}
$$

with poles at $n+(2 \pi i) /(\log q) \mathbb{Z}$. We note that $\left.\left\langle\mathcal{R}_{\alpha}, \Phi\right\rangle\right|_{\alpha=0}=\langle\delta, \Phi\rangle$, that is $1 /\left(\Gamma_{n}(0)\right)\|x\|_{K}^{-n}$ is equal to the Dirac delta function.

Proposition 2.1. ([17, Theorem 4.5]) As elements of $S^{\prime}\left(K^{n}\right)$,

$$
\mathcal{F}\left(\frac{\|x\|_{K}^{\alpha-n}}{\Gamma_{n}(\alpha)}\right)=\|x\|_{K}^{-\alpha}, \quad \alpha \notin n+\frac{2 \pi i}{\log q} \mathbb{Z} .
$$

2.2. IguSA'S LOCAL zetA function. Let $g(x) \in K[x], x=\left(x_{1}, \ldots, x_{n}\right)$, be a nonconstant polynomial, the $p$-adic complex power $|g|_{K}^{s}$ associated to $g$ (also called the Igusa local zeta function of $g$ ) is the distribution

$$
\left\langle|g|_{K}^{s}, \Phi\right\rangle=\int_{K^{n} \backslash g^{-1}(0)} \Phi(x)|g(x)|_{K}^{s}|d x|, s \in \mathbb{C}, \operatorname{Re}(s)>0 .
$$

For a fixed $\Phi$ we put $Z_{\Phi}(s, g)=\left\langle|g|_{K}^{s}, \Phi\right\rangle, \operatorname{Re}(s)>0$. In the case in which $\Phi$ is the characteristic function of $R_{K}^{n}$ we denote the local zeta function of $g$ by $Z(s, g)$. The local zeta functions were introduced by Weil $[20]$ and their basic properties for general $g$ were first studied by Igusa $[5,6]$.

A basic tool in the study of the local zeta functions is Hironaka's resolution Theorem [4]. This theorem guarantees the existence of an $n$-dimensional $K$-analytic manifold $Y$, a finite set $E=\{E\}$ of closed submanifolds of $Y$ of codimension 1 with a pair of positive integers $\left(N_{E}, n_{E}\right)$ assigned to each $E$, and a proper $K$-analytic map $h: Y \rightarrow K^{n}$ satisfying the following properties:

(I) $(g \circ h)^{-1}(0)=\bigcup_{E \in \mathcal{E}} E$;

(II) the restriction of $h$ to $Y \backslash h^{-1}\left(g^{-1}(0)\right)$ is an isomorphism onto its image; and

(III) at every point $b$ of $Y$ if $E_{1}, \ldots, E_{p}$ are all the $E$ in $\mathcal{E}$ containing $b$ with respective local equations $y_{1}, \ldots, y_{p}$ around $b$ and $\left(N_{i}, n_{i}\right)=\left(N_{E}, n_{E}\right)$ for $E=E_{i}$, 
then there exist local coordinates of $Y$ around $b$ of the form $\left(y_{1}, \ldots, y_{p}, y_{p+1}, \ldots, y_{n}\right)$ such that

$$
g \circ h=\varepsilon \prod_{1 \leqslant i \leqslant p} y_{i}^{N_{i}}
$$

on some neighbourhood of $b$, with $\varepsilon$ a unit of the local ring of $Y$ at $b$. The pair $(h, Y)$ is called a resolution of singularities for $g^{-1}(0)$, and $\bigcup_{E \in \mathcal{E}}\left\{\left(N_{E}, n_{E}\right)\right\}$ is the set of numerical data of $h$. A central result in the theory of local zeta functions is the following.

THEOREM 2.1. (Igusa, [5, Theorem 8.2.1]) Let $g(x) \in K[x]$ be a non-constant polynomial. The distribution $|g|_{K}^{s}$ admits a meromorphic continuation to the complex plane such that $\left\langle|g|_{K}^{s}, \Phi\right\rangle$ is a rational function of $q^{-s}$ for each $\Phi \in S\left(K^{n}\right)$. Furthermore, if $h: Y \rightarrow K^{n}$ is a resolution of singularities of $g^{-1}(0)$, with numerical data $\bigcup_{E \in \mathcal{E}}\left\{\left(N_{E}, n_{E}\right)\right\}$, then

$$
\prod_{E \in \mathcal{E}}\left(1-q^{-n_{E}-N_{E} s}\right)|g|_{K}^{s}
$$

is a holomorphic distribution. In particular the real parts of the poles of $|g|_{K}^{s}$ are negative rational numbers.

We shall denote by $|g|_{K}^{s}$ the meromorphic continuation of distribution (2.2), and by $Z(s, g)$, the integral $\int_{R_{K}^{n}}|g(x)|_{K}^{s}|d x|, \operatorname{Re}(s)>0$, and its meromorphic continuation to the complex plane. Theorem 2.1 is valid for distributions of the form $\chi(\operatorname{ac} g)|g|_{K}^{s}$, where $\chi$ is a multiplicative character of $R_{K}^{\times}$(see [5, Theorem 8.2.1]).

We set $\Omega_{l}, l \in \mathbb{Z}$, for the characteristic function of the ball $\left(P_{K}^{l}\right)^{n}$. We denote by $\Delta_{0}\left(K^{n}\right)$ the $\mathbb{C}$-vector space generated by $\Omega_{l}, l \in \mathbb{N}$, by $\Delta_{\infty}\left(K^{n}\right)$ the $\mathbb{C}$-vector space generated by $\Omega_{-l}, l \in \mathbb{N}$, and by $\Delta\left(K^{n}\right)$ the $\mathbb{C}$-vector space $\Delta_{0}\left(K^{n}\right) \oplus \Delta_{\infty}\left(K^{n}\right)$. The Fourier transform establishes a $\mathbb{C}$-isomorphism between $\Delta_{0}\left(K^{n}\right)$ and $\Delta_{\infty}\left(K^{n}\right)$, and therefore a $\mathbb{C}$-isomorphism from $\Delta\left(K^{n}\right)$ onto itself.

Lemma 2.1. Let $f(x) \in K[x]$ be a form of degree $d$. Then the distribution $|f|_{K}^{s}$ satisfies

$$
\left\langle|f|_{K}^{s}, \Phi\right\rangle=\left(\frac{1-q^{d s}}{1-q^{-n}}\right) Z(s, f)\left\langle\mathcal{R}_{d s+n}, \Phi\right\rangle,
$$

for $s \in \mathbb{C}$, and $\Phi \in \Delta\left(K^{n}\right)$.

Proof: Every $\Phi \in \Delta\left(K^{n}\right)$ is a finite linear combination of the form

$$
\Phi(x)=\sum_{i} c_{i} \Omega_{l_{i}}(x),
$$

where $c_{i} \in \mathbb{C}, l_{i} \in \mathbb{Z}$, and $\Omega_{l_{i}}$ is the characteristic function of the ball $\left(P_{K}^{l_{i}}\right)^{n}$.The action of $|f|_{K}^{s}$ on $\mathcal{F} \Phi$ can be explicitly described as follows:

$$
\left\langle|f|_{K}^{s}, \mathcal{F} \Phi\right\rangle=\sum_{i} c_{i}\left\langle|f|_{K}^{s}, q^{-n l_{i}} \Omega_{-l_{i}}\right\rangle
$$


and since

$$
\left\langle|f|_{K}^{s}, q^{-n l_{i}} \Omega_{-l_{i}}\right\rangle=q^{-n l_{i}} \int_{K^{n}}|f(x)|_{K}^{s} \Omega_{-l_{i}}(x)|d x|=Z(s, f) q^{d l_{i} s},
$$

for $\operatorname{Re}(s)>0$, it follows from (2.3) that

$$
\left\langle|f|_{K}^{s}, \mathcal{F} \Phi\right\rangle=Z(s, f) \sum_{i} c_{i} q^{d d_{i} s}, \text { for } \operatorname{Re}(s)>0 .
$$

On the other hand,

$$
\left\langle\frac{1-q^{d s}}{1-q^{-n}} \mathcal{R}_{d s+n}, q^{-n l_{i}} \Omega_{-l_{i}}\right\rangle=\left\langle\frac{1-q^{-n-d s}}{1-q^{-n}}\|x\|_{K}^{d s}, q^{-n l_{i}} \Omega_{-l_{i}}\right\rangle=q^{d l_{i} s},
$$

for every $l_{i} \in \mathbb{Z}$, and $\operatorname{Re}(s)>0$. Then (2.5) and (2.6) imply that

$$
\left\langle|f|_{K}^{s}, \mathcal{F} \Phi\right\rangle=\left(\frac{1-q^{d s}}{1-q^{-n}}\right) Z(s, f)\left\langle\mathcal{R}_{d s+n}, \mathcal{F} \Phi\right\rangle,
$$

for $\operatorname{Re}(s)>0$. By Theorem 2.1 and $(2.1),|f|_{K}^{s}, Z(s, f)$, and $\mathcal{R}_{d s+n}$ have a meromorphic continuation to the complex plane, therefore (2.7) extends to $\mathbb{C}$. Finally, since the Fourier transform establishes a $\mathbb{C}$-isomorphism from $\Delta\left(K^{n}\right)$ onto itself, it is possible to remove the Fourier transform symbol in (2.7).

THEOREM 2.2. Let $f(x) \in K[x]$ be a non-constant form of degree $d$. Then the distribution $|f|_{K}^{s}$ satisfies

$$
\left\langle|f|_{K}^{s}, \Phi\right\rangle=\frac{Z(s, f)}{Z(-s-(n / d), f)}\left\langle|f|_{K}^{-s-(n / d)}, \mathcal{F} \Phi\right\rangle, s \in \mathbb{C} \backslash\{0\}, \Phi \in \Delta\left(K^{n}\right) .
$$

ProOF: Suppose that $d s \notin-n+(2 \pi i / \log q) \mathbb{Z}$, by rewriting (2.7) as

$$
\left\langle|f|_{K}^{s}, \mathcal{F} \Phi\right\rangle=\left(\frac{1-q^{-n-d s}}{1-q^{-n}}\right) Z(s, f)\left\langle\|x\|_{K}^{d s}, \mathcal{F} \Phi\right\rangle, s \in \mathbb{C}
$$

and applying Proposition 2.1 we obtain that

$$
\left\langle|f|_{K}^{s}, \mathcal{F} \Phi\right\rangle=\left(\frac{1-q^{d s}}{1-q^{-n}}\right) Z(s, f)\left\langle\|x\|_{K}^{-d s-n}, \Phi\right\rangle,
$$

for $s \in \mathbb{C} \backslash\{-n+(2 \pi i / \log q) \mathbb{Z}\}$. By making $s \rightarrow-(s+(n / d))$ in $(2.10)$, it takes the following form:

$$
\left\langle|f|_{K}^{-s-(n / d)}, \mathcal{F} \Phi\right\rangle=\left(\frac{1-q^{-d s-n}}{1-q^{-n}}\right) Z\left(-s-\frac{n}{d}, f\right)\left\langle\|x\|_{K}^{d s}, \Phi\right\rangle,
$$

for $s \in \mathbb{C} \backslash\{(2 \pi i) /(\log q \mathbb{Z})\}$. By comparing (2.11) and (2.9), we obtain (2.8) for $s \in \mathbb{C} \backslash\{(2 \pi i) /(\log q) \mathbb{Z}\}$. In order to complete the proof, we have to show that (2.8) is 
valid for $s \in\{(2 \pi i) /(\log q) \mathbb{Z}\}$. We recall that $R_{0}=\delta$, that is, $1 / \Gamma_{n}(0)\|x\|_{K}^{-n}$ is equal to the Dirac delta function. In the case in which $d s+n=0 \bmod (2 \pi i / \log q) \mathbb{Z},(2.7)$ takes the form

$$
\begin{aligned}
\left\langle|f|_{K}^{-(n / d)}, \mathcal{F} \Phi\right\rangle & =Z(-(n / d), f)\left\langle\mathcal{R}_{0}, \mathcal{F} \Phi\right\rangle=Z(-(n / d), f)\langle\delta, \mathcal{F} \Phi\rangle \\
& =Z(-(n / d), f)\left\langle\mathcal{F}^{-1} \delta, \Phi\right\rangle=Z(-(n / d), f)\langle 1, \Phi\rangle
\end{aligned}
$$

On the other hand, since $|f|_{K}^{s}$ is holomorphic at zero, the Lebesgue Lemma implies that

$$
\left\langle|f|_{K}^{0}, \Phi\right\rangle=\lim _{s \rightarrow 0}\left\langle|f|_{K}^{s}, \Phi\right\rangle=\int_{K^{n}} \Phi(x)|d x|=\langle 1, \Phi\rangle
$$

In particular

$$
Z(0, f)=1
$$

Then from (2.12)-(2.14) follow that

$$
\left\langle|f|_{K}^{0}, \Phi\right\rangle=\langle 1, \Phi\rangle=\frac{Z(0, f)}{Z(-(n / d), f)}\left\langle|f|_{K}^{-(n / d)}, \mathcal{F} \Phi\right\rangle
$$

Therefore (2.8) is valid for every for $s \in\{(2 \pi i / \log q) \mathbb{Z}\}$.

We note that Lemma 2.1 and Theorem 2.2 are valid for distributions of the form

$$
\left\langle\chi(\operatorname{ac} f)|f|_{K}^{s}, \Phi\right\rangle=\int_{K^{n}} \Phi(x) \chi(\operatorname{ac} f(x))|f(x)|_{K}^{s}|d x| .
$$

Functional equations for distributions of type $\chi(\operatorname{ac} f)|f|_{K}^{s}$ have been obtain by Igusa [8], Sato [15], Gyoja [3], and Denef and Meuser [2].

\section{FUNDAMENTAL SOLUTIONS OF $p$-ADIC PSEUDO-DIFFERENTIAL EQUATIONS}

Given a polynomial function $f\left(x_{1}, \ldots, x_{n}\right)$ with coefficients in $K$ we define a pseudodifferential operator $f(\partial, \beta), \beta>0$, that acts on functions in $S\left(K^{n}\right)$ by

$$
\begin{aligned}
f(\partial, \beta) \Phi(x) & =\mathcal{F}^{-1}\left(|f|_{K}^{\beta} \mathcal{F} \Phi\right)(x) \\
& =\int_{K^{n} \backslash \mathcal{f}^{-1}(0)}|f(y)|_{K}^{\beta}(\mathcal{F} \Phi)(y) \Psi([x, y]) d y .
\end{aligned}
$$

Since $\left.|f|_{K}^{\beta} \mathcal{F} \Phi\right|_{K^{n} \backslash \mathcal{f}^{-1}(0)} \in S\left(K^{n}\right)$, and the Fourier transform is a linear isomorphism from $S\left(K^{n}\right)$ onto itself, it holds that $f(\partial, \beta) \Phi \in S\left(K^{n}\right)$. Thus $f(\partial, \beta)$ is a linear operator from $S\left(K^{n}\right)$ into $S\left(K^{n}\right)$. The operator $f(\partial, \beta)$ is continuous and has a self-adjoint extension 
with dense domain in $L^{2}\left(K^{n}\right)$. We associate to $f(\partial, \beta)$ the following $p$-adic pseudodifferential equation:

$$
f(\partial, \beta) u=g, g \in \mathcal{V} \subseteq \mathcal{S}\left(K^{n}\right) .
$$

A fundamental solution for (3.1) on $\mathcal{V}$ is a distribution $E_{\beta}$ such that $u=E_{\beta} * g$ is a solution. In a recent note [21] the author observed that Atiyah's proof of the existence of a fundamental solution for a differential operator with constant coefficients can be adapted to prove the existence of a fundamental solution for (3.1) on $\mathcal{S}\left(K^{n}\right)$.

The following Theorem describes explicitly a fundamental solution of (3.1) as a distribution on $\Delta\left(K^{n}\right)$ when $f(x)$ is a form of degree $d$ in $n$ variables.

TheOREM 3.1. Let $f(x) \in K\left[x_{1}, \ldots, x_{n}\right] \backslash K$ be a form of degree $d$, and $f(\partial, \beta)$ the $p$-adic pseudo-differential operator with symbol $|f|_{K}^{\beta}, \beta>0$. If

$$
-\beta \notin \bigcup_{E \in \mathcal{E}}\left\{-\frac{n_{E}}{N_{E}}\right\} \cup\left\{-\frac{n}{d}\right\} \cup\{\gamma \in \mathbb{R} \mid Z(\gamma, f)=0\},
$$

where $\bigcup_{E \in \mathcal{E}}\left\{\left(N_{E}, n_{E}\right)\right\}$ are the numerical data of a resolution of singularities $(Y, h)$ for $f^{-1}(0)$, then the distribution

$$
E_{\beta}(x)=\left(\frac{1-q^{-d \beta}}{1-q^{-n}}\right) Z(-\beta, f)\|x\|_{K}^{d \beta-n}
$$

is a fundamental solution of the $p$-adic pseudo-differential equation $f(\partial, \beta) u=g$, with $g \in \Delta\left(K^{n}\right)$.

Proof: We set $E_{\beta}=\mathcal{F}^{-1} T$, where $T \in S^{\prime}\left(K^{n}\right)$ satisfying $|f|_{K}^{\beta} T=1$. Then $E_{\beta}$ is a fundamental solution of (3.1). In order to prove the existence of $T$ we proceed as follows. By Theorem 2.1 the distribution $|f|_{K}^{s}$ has a meromorphic continuation to $\mathbb{C}$. Let

$$
|f|_{K}^{s}=\sum_{m \in \mathbf{Z}} T_{m}(s+\beta)^{m}
$$

be the Laurent expansion at $-\beta$ with $T_{m} \in S^{\prime}\left(K^{n}\right)$ for all $m$. Since the real parts of the poles of $|f|_{K}^{s}$ are negative rational numbers by Theorem $2.1,|f|_{K}^{s+\beta}=|f|_{K}^{\beta}|f|_{K}^{s}$ is holomorphic at $s=-\beta$. Therefore $|f|_{K}^{\beta} T_{m}=0$ for all $m<0$ and

$$
|f|_{K}^{s+\beta}=T_{0}|f|_{K}^{\beta}+\sum_{m=1}^{\infty} T_{m}|f|_{K}^{\beta}(s+\beta)^{m} .
$$

By using the Lebesgue Lemma and (3.3)

$$
\begin{aligned}
\lim _{s \rightarrow-\beta}\left\langle|f|_{K}^{s+\beta}, \Phi\right\rangle & =\int_{K^{n}} \Phi(x)|d x|=\langle 1, \Phi\rangle \\
& =T_{0}|f|_{K}^{\beta} .
\end{aligned}
$$


Therefore we can take $T=T_{0}$. Now since $\left\langle|f|_{K}^{s}, \Phi\right\rangle$ is a rational function of $q^{-s}$ for every $\Phi$ in $S\left(K^{n}\right)$, and $-\beta$ is not a pole of $|f|_{K}^{s}$ it holds that

$$
\left\langle T_{0}, \Phi\right\rangle=\lim _{s \rightarrow-\beta}\left\langle|f|_{K}^{s}, \Phi\right\rangle
$$

for every $\Phi$ in $S\left(K^{n}\right)$, that is,

$$
T_{0}=\lim _{s \rightarrow-\beta}|f|_{K}^{s} \ldots
$$

In order to find $E_{\beta}$, we compute explicitly $T_{0}$ by using (3.5), and then $E_{\beta}$ as $\mathcal{F}^{-1} T_{0}$. By Lemma 2.1

$$
|f|_{K}^{s}=\left(\frac{1-q^{-n-d s}}{1-q^{-n}}\right) Z(s, f)\|x\|_{K}^{d s}, s \in \mathbb{C},
$$

as a distribution on $\Delta\left(K^{n}\right)$. By using (3.5) we obtain from (3.6) that

$$
T_{0}=\left(\frac{1-q^{-n+\beta d}}{1-q^{-n}}\right) Z(-\beta, f)\|x\|_{K}^{-\beta d}
$$

as a distribution on $\Delta\left(K^{n}\right)$. If $\beta \neq n / d$, using Proposition (2.1) and the fact that the Fourier transform is an isomorphism on $\Delta\left(K^{n}\right)$, we obtain that $\mathcal{F}^{-1} T_{0}$ is equal to

$$
E_{\beta}(x)=\left(\frac{1-q^{-d \beta}}{1-q^{-n}}\right) Z(-\beta, f)\|x\|_{K}^{\beta d-n}, \text { if } \beta \neq \frac{n}{d},
$$

as a distribution on $\Delta\left(K^{n}\right)$. Finally, the condition $Z(-\beta, f) \neq 0$ implies $E_{\beta} \neq 0$.

3.1. REMARKs. Let $E$ be in $\mathcal{S}^{\prime}\left(K^{n}\right)$, and $g$ a complex valued function defined on $X \subseteq K^{n}$ having

$$
g(x)=\sum_{i=0}^{\infty} a_{k} \varphi_{k}(x), x \rightarrow x_{0}
$$

as an asymptotic expansion as $x$ tends to $x_{0}$, here $x_{0}$ is a limit point of $X$. If

$$
\langle E, \Phi\rangle=\int_{K^{n}} g(x) \Phi(x)|d x|
$$

for any $\Phi \in \mathcal{S}\left(K^{n}\right)$ whose support is contained in a sufficiently small ball around $x_{0}$, then we shall say that $E$ has an asymptotic expansion as $x$ tends to $x_{0}$, and write

$$
E(x)=\sum_{i=0}^{k} a_{k} \varphi_{k}(x)+O\left(\varphi_{k+1}(x)\right), x \rightarrow x_{0}
$$

Corollary 3.1. With the hypothesis of Theorem 3.1, it holds that (I) $E_{\beta}(x)$ $=O\left(\|x\|_{K}^{d \beta-n}\right)$ as $\|x\|_{K} \rightarrow 0$; (II) $E_{\beta}(x)=O\left(\|x\|_{K}^{-d \beta}\right)$ as $\|x\|_{K} \rightarrow \infty$. Moreover, the fundamental solution $E_{\beta}(x)$ is non-singular at the origin if $\beta>n / d$.

Proof: The first part follows directly from Theorem 3.1, and Remark 3.1 (3). The second part follows from the fact that

$$
\left\langle E_{\beta}(x), \Omega_{-\ell}\right\rangle=\left\langle E_{\beta}(x), \mathcal{F} \Omega_{\ell}\right\rangle=\left\langle\left(\frac{1-q^{d \beta-n}}{1-q^{-n}}\right) Z(-\beta, f)\|x\|_{K}^{-d \beta}, \Omega_{\ell}\right\rangle
$$


3.2. Elliptic PSEUdo-DifFERENTIAL OPERATORs. A quadratic form

$$
h\left(x_{1}, \ldots, x_{n}\right) \in \mathbb{Q}_{p}\left[x_{1}, \ldots, x_{n}\right], p \neq 2,
$$

is called elliptic (or anisotropic) if it satisfies

$$
h\left(x_{1}, \ldots, x_{n}\right) \neq 0 \text { if }\left|x_{1}\right|_{Q_{p}}+\ldots+\left|x_{n}\right|_{Q_{p}} \neq 0 .
$$

Other quadratic forms are called isotropic. A pseudo-differential operator $h(\partial, \beta)$ with symbol $|h|_{K}^{\beta}$ satisfying (3.8) is called an elliptic operator. It is well-known that there no exist anisotropic quadratic forms if $n \geqslant 5$. The following table shows all the anisotropic quadratic forms up to linear isomorphism.

\begin{tabular}{|c|c|}
\hline$n$ & Quadratic Forms \\
\hline 2 & $h\left(x_{1}, x_{2}\right)=x_{1}^{2}-\tau x_{2}^{2}, \tau \in \mathbb{Q}_{p} \backslash \mathbb{Q}_{p} \mathbb{Q}_{p}, \tau=\varepsilon, \tau=p, \tau=\varepsilon p, \varepsilon \in \mathbb{Z}_{p}^{\times}$ \\
\hline 3 & $h\left(x_{1}, x_{2}, x_{3}\right)=p \varepsilon_{1} x_{1}^{2}+\varepsilon_{2} x_{2}^{2}+\varepsilon_{3} x_{3}^{2}, \varepsilon_{1}, \varepsilon_{2}, \varepsilon_{3} \in \mathbb{Z}_{p}^{\times}$, \\
\hline 4 & $\varepsilon_{2} x_{2}^{2}+\varepsilon_{3} x_{3}^{2} \neq 0$ if $\left|x_{1}\right|_{\mathbb{Q}_{p}}+\left|x_{2}\right|_{\mathbb{Q}_{p}} \neq 0$ \\
\hline & $h\left(x_{1}, x_{2}, x_{3}, x_{4}\right)=x_{1}^{2}-s x_{2}^{2}-p x_{3}^{2}+s p x_{4}^{2}, s \in \mathbb{Z}$, with $(s / p)=-1$ \\
\hline
\end{tabular}

Our next goal is to determine a fundamental solution for an elliptic operator on $\Delta\left(K^{n}\right)$. The first step is to calculate $Z(s, h)$. This calculation can be easily accomplished by using the $p$-adic stationary phase formula. This method introduced by Igusa [7] permits the calculation of the local zeta function for a wide class of polynomials $[\mathbf{7}$, and the references therein], $[\mathbf{2 2 ,} \mathbf{2 3}, \mathbf{2 4}]$.

If $h(x) \in R_{K}\left[x_{1}, \ldots, x_{n}\right] \backslash \mathfrak{p} R_{K}\left[x_{1}, \ldots, x_{n}\right]$, we denote by $\bar{h}(x)$ its reduction modulo $\mathfrak{p} R_{K}$.

Proposition 3.1. ([5, Proposition 10.2.1]) If

$$
h(x) \in R_{K}\left[x_{1}, \ldots, x_{n}\right] \backslash \mathfrak{p} R_{K}\left[x_{1}, \ldots, x_{n}\right]
$$

is a homogeneous polynomial of degree $d$ such that $\bar{h}(\bar{a})=\frac{\partial \bar{h}}{\partial x_{i}}(\bar{a})=0$ for $1 \leqslant i \leqslant n$ implies $\bar{a}=0$, then

$$
Z(s, h)=\frac{\left(1-q^{-n} N\right)+\left(q^{-n-1}+q^{-n}(N-1)-q^{-1}\right) q^{-s}}{\left(1-q^{-1-s}\right)\left(1-q^{-n-d s}\right)}
$$

where $N$ denotes the number of zeros of $\bar{f}(x)$ in $\mathbb{F}_{q}^{n}$.

We shall identify an elliptic quadratic form with one of the polynomials listed in table (3.9). Then as a consequence of the previous Proposition we obtain the following result.

COROLlaRY 3.2. If $h(x) \in \mathbb{Z}_{p}[x]$ is an elliptic quadratic form in $n$ variables, then $Z(s, h)=\left(1-q^{-n}\right) /\left(1-q^{-n-2 s}\right)$. 
The following result describes explicitly a fundamental solution of $h(\partial, \beta) u=g$, $g \in \Delta\left(K^{n}\right)$; this result follows from Theorem 3.1 and Corollary 3.2.

THEOREM 3.2. Let $h(x) \in \mathbb{Z}_{p}\left[x_{1}, \ldots, x_{n}\right]$ be an elliptic quadratic form, and $h(\partial, \beta)$ the $p$-adic pseudo-differential operator with symbol $|h|_{K}^{\beta}, \beta>0$. If $\beta \neq n / 2$, then the distribution

$$
E_{\beta}(x)=\left(\frac{1-p^{-2 \beta}}{1-p^{-n+2 \beta}}\right)\|x\|_{\mathbb{Q}_{p}}^{2 \beta-n}
$$

is a fundamental solution of the $p$-adic pseudo-differential equation $h(\partial, \beta) u=g$, with $g \in \Delta\left(K^{n}\right)$.

Theorem 3.2 is valid for any finite extension $K$ of $\mathbb{Q}_{p}$. In [12], [11, Chapter 2] Kochubei calculated explicitly the fundamental solutions for the elliptic operators. The restriction of these distributions to the space $\Delta\left(K^{n}\right)$ coincide with the distributions given in Theorem 3.2 when $\beta \neq n / 2$.

\section{4. p-Adic Green Functions}

A fundamental solution $G_{\lambda}$ of the pseudo-differential

$$
(f(\partial, \beta)+\lambda) u=g, \quad \beta, \lambda \in \mathbb{R}, \beta, \lambda>0, g \in \mathcal{S}\left(K^{n}\right)
$$

is called a $p$-adic Green function. Since $|f(x)|_{K}^{\beta}+\lambda \neq 0$, for every $x$ in $K^{n}$, the distribution

$$
G_{\lambda}=\mathcal{F}^{-1}\left(\frac{1}{|f|_{K}^{\beta}+\lambda}\right)
$$

is a Green function. We shall say that $G_{\lambda}$ is the Green function associated to the operator $f(\partial, \beta)$.

4.1. INTEGRATION ON THE FIBERS. Let $g(x)$ be a non-constant polynomial with coefficients in $K$. Let $C_{g}$ and $S_{g}=g\left(C_{g}\right)$ denote the set of critical points and the set of critical values of the mapping $g: K^{n} \rightarrow K$. In the case in which $g$ a homogeneous polynomial $S_{g}=\{0\}$ (see for example [5, Theoren 2.5.1]). For any $z \in K^{n} \backslash\{0\}$ we define $|d x / d g|$ to be the residue of the measure $|d x|$ along the fiber $g^{-1}(z)$, and

$$
F_{\Phi}(z)=\int_{g^{-1}(z)} \Phi(x)\left|\frac{d x}{d g}\right|,
$$

where $\Phi \in \mathcal{S}\left(K^{n}\right)$ is a fixed function. If $\Phi$ is the characteristic function of $R_{K}^{n}$ we use $F(z)$ instead of $F_{\Phi}(z)$. The function $F_{\Phi}(z)$ is locally constant on $K \backslash\{0\}$, and satisfies

$$
\int_{K^{n}} \Phi(z)|d z|=\int_{K \backslash\{0\}} F_{\Phi}(z)|d z|
$$

for every $\Phi$ in $\mathcal{S}\left(K^{n}\right)$ (see [5, Lemma 8.3.2]). The following Lemma, that follows from (4.2), will be used later on. 
LEMMA 4.1. (See [5, Theorem 8.4.1].) Let $g(x) \in K\left[x_{1}, \ldots, x_{n}\right] \backslash K$ be a nonconstant polynomial such that $C_{g}$ is contained in $g^{-1}(0)$. Then

$$
Z_{\Phi}(s, g)=\int_{K \backslash\{0\}} F_{\Phi}(z)|z|_{K}^{s}|d z|, \quad \operatorname{Re}(s)>0 .
$$

4.2. AsYMPTOTICS OF $G_{\lambda}(x)$ AS $\|x\|_{K} \rightarrow \infty$.

Theorem 4.1. Let $f(x) \in R_{K}\left[x_{1}, \ldots, x_{n}\right] \backslash K$ be a form of degree $d$, and $f(\partial, \beta)$ the $p$-adic pseudo-differential operator with symbol $|f|_{K}^{\beta}, \beta>0$. The Green function $G_{\lambda}$ corresponding to $f(\partial, \beta)$ admits the asymptotic expansion

$$
G_{\lambda}(x)=\left(\frac{1}{1-q^{-n}}\right) \sum_{m=1}^{\infty} \frac{(-1)^{m-1}\left(1-q^{d \beta m}\right)}{\lambda^{m+1}} Z(\beta m, f)\|x\|_{K}^{-d \beta m-n}
$$

as $\|x\|_{K} \rightarrow \infty$. In particular $G_{\lambda}(x)$ satisfies

$$
G_{\lambda}(x)=\left(\frac{1}{1-q^{-n}}\right) \frac{\left(1-q^{d \beta}\right)}{\lambda^{2}} Z(\beta, f)\|x\|_{K}^{-d \beta-n}+O\left(\frac{1}{\lambda^{3}}\|x\|_{K}^{-2 d \beta-n}\right)
$$

as $\|x\|_{K} \rightarrow \infty$.

Proof: The asymptotic behaviour of $G_{\lambda}$ at infinity can be studied by considering the action of $G_{\lambda}$ on functions of type $\Omega_{-l}$, the characteristic function of the ball $\left(P_{K}^{-l}\right)^{n}$, as $l \rightarrow \infty$ (see Remark 3.1). By definition

$$
\begin{aligned}
\left\langle G_{\lambda}, \Omega_{-l}\right\rangle & =\left\langle\frac{1}{|f|_{K}^{\beta}+\lambda}, \mathcal{F}^{-1} \Omega_{-l}\right\rangle=\left\langle\frac{1}{|f|_{K}^{\beta}+\lambda}, q^{n l} \Omega_{l}\right\rangle \\
& =\int_{R_{K}^{n}} \frac{|d z|}{q^{-l d \beta}|f(z)|_{K}^{\beta}+\lambda},
\end{aligned}
$$

and by using integration on the fibers, (4.4) can be rewritten as

$$
\left\langle G_{\lambda}, \Omega_{-l}\right\rangle=\int_{R_{K} \backslash\{0\}} \frac{F(t)}{q^{-l d \beta}|t|_{K}^{\beta}+\lambda}|d t|
$$

By applying the asymptotic expansion

$$
\frac{1}{1+y}=\sum_{m=1}^{\infty}(-1)^{m-1} y^{n}, \text { as } y \rightarrow 0
$$

in (4.5) we obtain the following asymptotic expansion for $\left\langle G_{\lambda}, \Omega_{-l}\right\rangle$ :

$$
\left\langle G_{\lambda}, \Omega_{-l}\right\rangle=\sum_{m=1}^{\infty}(-1)^{m-1} \frac{q^{-l d \beta m}}{\lambda^{m+1}} \int_{R_{K} \backslash\{0\}}|t|_{K}^{\beta m} F(t)|d t|, l \rightarrow \infty .
$$


By using Lemma 4.1 and (2.6) in (4.6), we obtain that

$$
\begin{aligned}
\left\langle G_{\lambda}, \Omega_{-l}\right\rangle & =\sum_{m=1}^{\infty}(-1)^{m-1} \frac{q^{-l d \beta m}}{\lambda^{m+1}} Z(\beta m, f) \\
& =\sum_{m=1}^{\infty}(-1)^{m-1} \frac{Z(\beta m, f)}{\lambda^{m+1}}\left\langle\frac{1-q^{-n-d \beta m}}{1-q^{-n}}\|x\|_{K}^{d \beta m}, q^{n l} \Omega_{-l}\right\rangle,
\end{aligned}
$$

as $l \rightarrow \infty$, that is,

$$
\left\langle G_{\lambda}, \Omega_{-l}\right\rangle=\sum_{m=1}^{\infty}(-1)^{m-1} \frac{Z(\beta m, f)}{\lambda^{m+1}}\left\langle\mathcal{F}^{-1}\left(\frac{1-q^{-n-d \beta m}}{1-q^{-n}}\|x\|_{K}^{d \beta m}\right), \Omega_{-l}\right\rangle .
$$

From (4.7) by using Proposition 2.1 we obtain that

$$
G_{\lambda}(x)=\sum_{m=1}^{\infty}(-1)^{m-1} \frac{\left(1-q^{d \beta m} f\right) Z(\beta m, f)}{\left(1-q^{-n}\right) \lambda^{m+1}}\|x\|_{K}^{-d \beta m-n},\|x\|_{K} \rightarrow \infty
$$

The second part follows directly from (4.8).

\subsection{REMARKS.}

1. The previous Theorem is valid for a twisted operator $f(\partial, \beta, \chi)$, in this case it is necessary to change $Z(\beta m, f)$ by $Z(\beta m, \chi, f)$ in the statement of Theorem 4.1.

2. 2. Kochubei has studied the asymptotics of the Green functions associated with elliptic operators (see for example, [11, Section 2.8]) at infinity and the origin. The asymptotics obtained by Kochubei at infinity can be recovered from Theorem 4.1.

3. Sato's asymptotic expansion for $G_{\lambda},[\mathbf{1 6}]$, is only valid for forms that are invariants of prehomogeneous vector spaces.

\section{REFERENCES}

[1] M.F. Atiyah, 'Resolution of singularities and division of distributions', Comm. Pure Appl. Math. 23 (1970), 145-150.

[2] J. Denef and D. Meuser, 'A functional equation of Igusa's local zeta function', Amer. $J$. Math. 109 (1987), 991-1008.

[3] A. Gyoja, 'Lectures on the functional equation satisfied by the $p$-adic local zeta functions of reductive prehomogeneous vector spaces', (unpublished) Lectures at the Johns Hopkins University.

[4] H. Hironaka, 'Resolution of singularities of an algebraic variety over a field of characteristic zero', Ann. Math. 79 (1964), 109-326.

[5] J.-I. Igusa, An introduction to the theory of local zeta functions, AMS/IP Studies in Advanced Mathematics 14 (American Mathematical Society, Providence R.I., 2000). 
[6] J.-I Igusa, 'Complex powers and asymptotic expansions', J, Reine Angew. Math. 268/269 (1974), 110-130. II, ibid., 278/279 (1975), 357-368.

[7] J.-I. Igusa, 'A stationary phase formula for $\mathrm{p}$-adic integrals and its applications', in $\mathrm{Al}$ gebraic Geometry and its Applications (Springer-Verlag, Berlin, Heidelberg, New York, 1994), pp. 175-194.

[8] J.-I. Igusa, 'Some results on p-adic complex powers', Amer. J. Math. 106 (1984), 1013-1032.

[9] Y. Jang, 'An asymptotic expansion of the p-adic Green function', Tohoku Math. J. 50 (1998), 229-242.

[10] A. Khrennikov, 'Fundamental solutions over the field of $p$-adic numbers', St. Peterburgh Math. J. 4 (1993), 613-628.

[11] A.N. Kochubei, Pseudodifferential equations and stochastics over non-archimedean fields (Marcel Dekker, New York, 2001).

[12] A.N. Kochubei, 'Fundamental solutions of pseudo-differential equations associated with p-adic quadratic forms', Izv. Math. 62 (1998), 1169-1188.

[13] A.N. Kochubei, 'On p-adic Green functions', Theoret. and Math. Phys. 96 (1993), 854-865.

[14] A.N. Kochubei, 'on the asymptotics of $p$-adic Green functions', Proc. Steklov Inst. Math. 203 (1994/95), 105-113.

[15] F. Sato, 'On functional equations of zeta distributions', Adv. Stud. Pure Math. 15 (1989), 465-508.

[16] F. Sato, 'p-adic Green functions and zeta functions', Comment. Math. Univ. St. Pauli 51 (2002), 79-97.

[17] M.H. Taibleson, Fourier analysis on local fields, Mathematical Notes 15 (Princeton University Press, Princeton, N.J., 1975).

[18] V.S. Vladimirov, 'On the spectrum of some pseudo-differential operators over $p$-adic number field', (in Russian), Algebra i Analiz 2 (1990), 107-124.

[19] V.S. Vladimirov, I.V. Volovich and E.I. Zelenov, p-adic analysis and mathematical physics (World Scientific, Singapore, 1994).

[20] A. Weil, 'Sur la formule de Siegel dans le théorie des groupes classiques', Acta Math. 113 (1965), 1-87.

[21] W.A. Zuniga-Galindo, 'Fundamental solutions of pseudo-differential operators over p-adic fields', Rend. Sem. Mat. Univ. Padova 109 (2003), 241-245.

[22] W.A. Zuniga-Galindo, 'Igusa's local zeta functions of semiquasihomogeneous polynomials', Trans. Amer. Math. Soc. 353 (2001), 3193-3207.

[23] W.A. Zuniga-Galindo, 'Local zeta functions and Newton Polyhedra', Nagoya Math. $J$. 172 (2003), 31-58.

[24] W.A. Zuniga-Galindo, 'Local zeta function for non-degenerate homogeneous mappings', Pacific J. Math. (to appear).

Department of Mathematics and Computer Science

Barry University

11300 N.E.Second Avenue

Miami Shores, FL 33161

United States of America

e-mail: wzuniga@mail.barry.edu 